\title{
Inteligencia cultural en la universidad: estrategias didácticas innovadoras aplicadas en el Centro Hispano-Ruso de la Universidad Federal del Sur, Rostov del Don, Rusia
}

\author{
Canese de Estigarribia, Marta Isabel \\ Inteligencia cultural en la universidad: estrategias didácticas innovadoras aplicadas en el Centro Hispano-Ruso de la \\ Universidad Federal del Sur, Rostov del Don, Rusia \\ CIENCIA ergo-sum, vol. 25, núm. 2, julio-octubre 2018|e12 \\ Universidad Autónoma del Estado de México, México \\ Esta obra está bajo una Licencia Creative Commons Atribución-NoComercial-SinDerivar 4.0 Internacional.
}

Canese de Estigarribia, M. I. (2018). Inteligencia cultural en la universidad: estrategias didácticas innovadoras aplicadas en el Centro Hispano-Ruso de la Universidad Federal del Sur, Rostov del Don, Rusia. CIENCIA ergo-sum, 25(2). https://doi.org/10.30878/ces.v25n2a2 
Inteligencia cultural en la universidad: estrategias didácticas innovadoras aplicadas en el Centro Hispano-Ruso de la Universidad Federal del Sur, Rostov del Don, Rusia

\author{
Cultural Intelligence at the University: Innovative Methodological Strategies Applied at the Southern Federal \\ University's Hispanic-Russian Center in Rostov on Don, Russia
}

Marta Isabel Canese de Estigarribia

Universidad del Norte, Paraguay, Paraguay

mcanese@gmail.com

Recepción: 31 de enero de 2017

Aprobación: 25 de mayo de 2017

\title{
RESUMEN:
}

Se identifican estrategias didácticas innovadoras para el desarrollo de la inteligencia cultural en el Centro Hispano-Ruso de la Universidad Federal del Sur, Federación Rusa. La metodología tiene un enfoque cualitativo, alcance exploratorio y corte transversal. Se aplicó mediante observaciones de actividades académicas y cuestionarios que respondieron docentes y alumnos entre septiembre y octubre de 2016. Los resultados permiten identificar cinco estrategias didácticas: enfoque comunicativo, motivación, autonomía del estudiante, aprendizaje colaborativo y construcción colectiva contextualizada, las cuales contribuyen al desarrollo de la inteligencia cultural y a la construcción de una cultura favorable a la comunicación intercultural, además de servir como referencia en el diseño de estrategias que promuevan una cultura internacional en universidades latinoamericanas.

PALABRAS CLAVE: enseñanza, estrategias, intercultural, integración, comunicación.

\begin{abstract}
:
The main objective of this study was to identify the methodological strategies implemented to develop cultural intelligence at the Southern Federal University's Hispanic-Russian Center in Rostov on Don, Russian Federation. This was a crosssectional exploratory study following a qualitative approach with observations and questionnaires with teachers and students during September and October 2016. Five methodological strategies were identified: communicative approach, motivation, student's autonomy, collaborative learning and contextualized collective construction. These strategies contribute to the development of cultural intelligence as well as the social construction of an institutional culture favorable to intercultural communication, and they can serve as a reference for the design of methodological strategies, to promote international culture at Latin American universities.
\end{abstract}

KEYWORDS: teaching methodology, intercultural, learning, communication, autonomy.

\section{INTRODUCCIÓN}

La inteligencia cultural constituye la base de la construcción del conocimiento humano y se encuentra implícita en la práctica educativa de las universidades. La inteligencia cultural la definen por Earley y Mosakowski (2004) como la inteligencia que permite comprender e interpretar el comportamiento, las emociones y los significados de una comunidad cultural diferente a la propia. En las primeras universidades de la Edad Media, los intercambios de estudiantes y docentes eran prácticas habituales, facilitadas gracias al uso de una lengua común en las actividades académicas y las bibliotecas: el latín. En la actualidad, la inteligencia cultural es un tema emergente en la investigación educativa, en todos los niveles y modalidades de la enseñanza, y constituye una prioridad en el diseño de las estrategias de enseñanza-aprendizaje.

Las universidades de Paraguay plantean, entre sus prioridades, la internacionalización de los estudios universitarios (Canese, 2010). Sin embargo, no cuentan con estudios sobre el diseño de estrategias didácticas para el desarrollo de la inteligencia cultural. Y son escasos los antecedentes de estudios específicos sobre el desarrollo de la inteligencia cultural en otros países de la región latinoamericana, entre los cuales se puede mencionar el de Domingo Depaula y Celeste Azzollini (2012) sobre la inteligencia cultural, valores 
y motivación para el aprendizaje en estudiantes militares argentinos. Esta carencia de estudios y modelos nacionales o regionales impulsó la búsqueda de estrategias didácticas en un centro de estudios e investigación dedicado a la comunicación intercultural con países hispanohablantes: el Centro Hispano-Ruso (CHR) de la Universidad Federal del Sur (UFS) de la ciudad Rostov del Don, Federación Rusa, con la finalidad de contribuir al diseño de estrategias didácticas para el desarrollo de la inteligencia cultural en las universidades de Paraguay.

El objetivo general de este estudio fue identificar las estrategias didácticas orientadas al desarrollo de la inteligencia cultural, aplicadas en el Centro Hispano-Ruso de la Universidad Federal del Sur, Federación Rusa. Los objetivos específicos fueron los siguientes: a) caracterizar las estrategias didácticas aplicadas para el desarrollo de la inteligencia cultural en el Centro Hispano-Ruso, $b$ ) clasificar las actividades didácticas implementadas para promover el desarrollo de la inteligencia cultural en el Centro Hispano-Ruso y $c$ ) determinar la percepción de los estudiantes y los profesores del Centro Hispano-Ruso sobre los aportes de las estrategias y actividades didácticas implementadas en el desarrollo de la inteligencia cultural.

La investigación se fundamentó en los aportes teóricos de Vigotsky y Luria (2007), de Bruner (2012) y de Esteban-Guitart et al. (2013), quienes consideran la inteligencia cultural como el elemento que distingue a la especie humana de las demás, dado que amplía la potencia intelectual de la mente humana más allá de las posibilidades individuales. Vigotsky y Luria (2007) afirman que las nuevas generaciones reciben la cultura construida en experiencias vividas por las generaciones anteriores y también los instrumentos y métodos que guían la conducta cultural. Concordando con estas afirmaciones, Bruner (2012) señala que el ser humano logra avanzar más de lo que permiten sus límites biológicos mediante la cultura o intercambiando signos, símbolos y tecnologías. Esteban-Guitart et al. (2013) agregan que, mediante elintercambio de un conjunto de conceptos, artefactos, instituciones sociales, prácticas, símbolos y significados, la cultura organiza la mente y la conducta humana. Para Esteban-Guitart (2012), el desarrollo cultural se explica mediante la intencionalidad compartida, la capacidad de comprender y compartir objetivos, experiencias y actividades cooperativas.

El concepto de estrategia didáctica, concordando con Mora (2010: 22), se define como el conjunto de "los procedimientos (métodos, técnicas, actividades) por los cuales el docente y los estudiantes organizan las acciones de manera consciente para construir y lograr metas previstas e imprevistas en el proceso de enseñanza y aprendizaje". El diseño de estrategias didácticas, según la misma autora, representa un modelo integrado de los procedimientos que facilitan al alumno la construcción de nuevos conocimientos.

El estudio desarrollado por Sémikova et al. (2015) señala la importancia del enfoque interdisciplinario y comunicativo en el diseño de estrategias didácticas. Estos autores han desarrollado una metodología innovadora, denominada galaxia espiral, que utiliza la intuición como primer acercamiento al conocimiento y desarrollo de las habilidades interculturales. Esta metodología promueve un estilo de aprendizaje ascendente en el que se va estudiando de forma progresiva el mismo fenómeno y aumenta la profundidad del conocimiento en cada etapa. Se aplica en ella el concepto de Zona de Desarrollo Proximal, de Lev Vigotsky (1979), definida como el conjunto de los conocimientos y las habilidades que el alumno puede adquirir, a partir de lo que ya conoce (Zona de Desarrollo Real). La metodología innovadora galaxia espiral fue laureada con el premio nacional Educación Global en 2012.

Los estudios de Earley y Ang (2003) delinean cuatro dimensiones en el proceso de desarrollo de la inteligencia cultural que constituyen la base de la elaboración de escalas para su medición: $a$ ) dimensión metacognitiva, $b$ ) dimensión cognitiva, $c$ ) dimensión motivacional y d) dimensión conductual. a) La dimensión metacognitiva comprende las capacidades mentales necesarias para el procesamiento eficiente de las interacciones interculturales, como la planificación, la conciencia y el monitoreo. $b$ ) La dimensión cognitiva comprende la adquisición de conocimientos culturales. Incluye tanto el conocimiento general de la cultura universal, como los conocimientos específicos de la cultura propia y la de los otros.c) La dimensión motivacional dinamiza todas las otras incrementando la atención y la colaboración con personas de otras culturas. Comprende el interés intrínseco o personal, el interés extrínseco o socio-cultural y la autoconfianza 
en el propio desempeño. d) La dimensión conductual consiste en la capacidad de seleccionar y utilizar las acciones comunicativas verbales y no verbales adecuadas para cada situación intercultural. Comprende a la conducta verbal, la conducta no verbal y la actuación comunicativa (Van Dyne et al., 2012).

\section{Materiales y MÉTODos}

Para alcanzar los objetivos propuestos, se realizó un estudio de caso en el Centro Hispano-Ruso de la Universidad Federal del Sur, Rostov del Don, Rusia, durante los meses de septiembre y octubre de 2016. La metodología aplicada tuvo un nivel exploratorio, con enfoque cualitativo y corte transversal. Las técnicas e instrumentos aplicados fueron $a$ ) la observación no participante y participante de las actividades didácticas, con registro de datos en un diario de campo y $b$ ) la aplicación de cuestionarios con preguntas abiertas a los profesores y estudiantes del centro que participaron en las actividades observadas.

Durante los meses de septiembre y octubre de 2016, se observaron un total de 11 actividades didácticas. Siete de ellas fueron observaciones participantes: cuatro conferencias, una videoconferencia y dos actividades de acompañamiento a visitantes extranjeros. Las cuatro observaciones no participantes fueron una observación de clase, dos observaciones de actividades de lectura en la biblioteca y una actividad de extensión universitaria. Los datos recogidos se registraron en un diario de campo para su posterior análisis.

El Centro Hispano-Ruso contaba, en el período de septiembre a octubre de 2016, con un plantel de 12 docentes y con 170 alumnos matriculados. El muestreo fue de tipo no probabilístico o dirigido, del tipo muestra de expertos (Hernández Sampieri et al., 2014), de modo que se contara con la opinión de informantes con mejor nivel de conocimiento sobre las estrategias didácticas innovadoras aplicadas en el Centro Hispano-Ruso. Si bien este tipo de muestra no es representativa al punto de poder generalizar los resultados obtenidos para la población estudiada, dadas las limitaciones de este tipo de muestreo, su aplicación en estudios cualitativos permite una aproximación y una mejor comprensión del fenómeno investigado. Los participantes en esta investigación fueron tres docentes investigadores, 14 estudiantes del último curso de la licenciatura en Filología y 13 estudiantes de la Maestría en Teoría de la Traducción y la Comunicación Intercultural.

Para determinar el número de sujetos participantes, se tomaron en cuenta los criterios establecidos por Hernández Sampieri et al. (2014): a) capacidad operativa, la cual considera que la investigación debe realizarse en un espacio de tiempo limitado, $b$ ) cantidad suficiente de sujetos para obtener los datos requeridos por la investigación y $c$ ) naturaleza del fenómeno estudiado en cuanto a su frecuencia y accesibilidad. Se establecieron dos unidades de análisis: docentes y alumnos. Para cada unidad de análisis, se identificaron los criterios de selección de los individuos, y así obtener la información necesaria para alcanzar los objetivos de la investigación.

Los criterios de inclusión de los docentes participantes son los siguientes: a) fungir como docente del último curso de la Licenciatura en Filología y de la Maestría en Teoría de la Traducción y la Comunicación Intercultural, en el período de setiembre a octubre de 2016, b) contar con investigaciones desarrolladas y publicadas sobre estrategias didácticas innovadoras y c) participar en la ejecución de las actividades observadas en el Centro Hispano-Ruso.

De los 12 profesores del centro, tres de los que participaron en el estudio cumplen estos criterios. A continuación los criterios correspondientes a los estudiantes participantes: a) estar matriculado como estudiante del último año de la Licenciatura en Filología, o en la Maestría en Teoría de la Traducción y la Comunicación Intercultural, en el período de setiembre a octubre de 2016. Este criterio obedece a que estos son los estudiantes que poseen mayor grado de conocimiento sobre las estrategias didácticas innovadoras en las que han participado durante sus estudios y $b$ ) participar en las actividades observadas por esta investigación. Como las observaciones se realizaron en días y horarios de clases obligatorias para los estudiantes, casi todos los alumnos de los cursos mencionados participaron. 
Los testimonios aportados por los sujetos participantes y los registros de las observaciones se analizaron mediante la técnica de análisis de contenido. Los datos se ordenaron en cuatro categorías: a) contexto institucional, $b$ ) estrategias, $c$ ) actividades y $d$ ) aportes al desarrollo de la inteligencia cultural. Esta última categoría, a su vez, comprendió cuatro subcategorías correspondientes a las dimensiones de la inteligencia cultural identificadas por Earley y Ang (2003): a) metacognitiva, b) cognitiva, $c$ ) motivacional y $d$ ) actitudinal.

\section{Resultados}

Los resultados obtenidos en esta investigación se organizaron en cuatro categorías de análisis: a) contexto institucional, $b$ ) estrategias didácticas, $c$ ) actividades didácticas y $d$ ) percepciones de los estudiantes y los profesores del Centro Hispano-Ruso sobre los aportes de las estrategias didácticas para el desarrollo de la inteligencia cultural. Los testimonios de los alumnos y los docentes, citados en el análisis de los resultados, son identificados por una letra (A para identificar a los alumnos y D para identificar a los profesores), seguida de un número que identifica a cada individuo manteniendo la confidencialidad de su identidad.

\subsection{Contexto institucional}

La Universidad Federal del Sur es el centro académico, científico y cultural más importante y de mayores dimensiones del sur de la Federación Rusa, y una de las universidades federales más antiguas. Sus antecedentes se remontan a la creación de la Universidad Imperial de Varsovia, fundada en 1816, con el propósito de proveer una educación superior a los militares rusos de Varsovia. En Rostov del Don, la universidad comenzó a funcionar desde 1915 como una filial de dicha universidad. Contaba con cuatro departamentos: historia y filosofía, medicina, física y matemáticas, y derecho.

En la actualidad, la Universidad Federal del Sur declara ser una universidad innovadora que promueve la investigación científica y la educación integral, además de formar a expertos altamente cualificados en los diversos campos de la ciencia. Caracterizada por su apertura intercultural, cuenta en la actualidad con más de 7000 profesionales graduados de 120 países. Está estructurada en diversas facultades e institutos, en las que abarca múltiples áreas del conocimiento: Facultad de Biología y Ciencias del Suelo, Facultad de Química, Facultad de Economía, Facultad de Geología y Geografía, Facultad de Alta Tecnología, Facultad de Historia, Facultad de Derecho, Facultad de Matemáticas, Mecánica y Computación, Facultad de Filología y Periodismo, Facultad de Filosofía y Estudios Culturales, Facultad de Psicología, Facultad de Física, Facultad de Estudios Regionales, Facultad de Ciencia Social y Política, Instituto de Relaciones Económicas Internacionales, Instituto Pedagógico, entre otras.

El Centro Hispano-Ruso de la Universidad Federal del Sur forma profesionales graduados en Filología y Periodismo y posgraduados en Comunicación Intercultural, con énfasis en cultura hispanoamericana. La misión de dicho centro es contribuir al diálogo intercultural de Rusia con los pueblos iberoamericanos a través del estudio de la lengua, la cultura y la civilización hispánica y latinoamericana. Esta misión se sustenta en tres ejes estratégicos: $a$ ) promover el estudio de la lengua y la cultura hispánica en sus diversas variaciones, b) promover la movilidad académica de estudiantes y profesores y $c$ ) gestionar vínculos con universidades de América Latina y España. Cuenta con un calificado cuerpo de docentes investigadores, con conocimientos específicos de la lengua española, la interpretación, la traducción y también conocimientos avanzados en el área pedagógica, didáctica y cultural. Se encuentra ubicado en el centro histórico de la ciudad de Rostov del Don, que cuenta con un importante centro industrial y cultural (Centro Hispano-Ruso, 2016). 


\section{2. Estrategias didácticas orientadas al desarrollo de la inteligencia cultural}

Las estrategias didácticas observadas en el Centro Hispano-Ruso se clasificaron en cinco tipos:

a) Estrategia de aprendizaje comunicativo: el aprendizaje se desarrolla en distintas situaciones de interacción comunicativa intercultural y son analizadas de forma simultánea. Las clases se imparten en idioma español desde el principio, en sus inicios con un vocabulario reducido (menor variedad de vocablos), construcción gramatical simple y verbalización más lenta. La complejidad gramatical, la variedad de vocablos y la velocidad de la verbalización incrementan a medida que los estudiantes adquieren los conocimientos y las habilidades necesarias para la comunicación fluida. El aprendizaje se centra en el desarrollo de competencias receptivas y productivas: el estudiante es animado a investigar y debatir, por lo que desarrolla al mismo tiempo el pensamiento abstracto, lógico y crítico y la capacidad de comprender las características de la cultura iberoamericana.

Durante las clases y actividades académicas observadas se registró una gran diversidad de situaciones de interacción comunicativa intercultural. Los estudiantes participaron activamente en los debates preguntando, formulando críticas o manifestando sus opiniones. Las respuestas de los estudiantes al cuestionario aplicado manifiestan que "el mejor momento de las charlas interactivas es cuando podemos hacer preguntas y manifestar nuestras opiniones" (A1).

b) Estrategia de motivación del estudiante: en el proceso de enseñanza-aprendizaje es decisiva la motivación personal del estudiante. Esto se logra mediante proyectos colaborativos, charlas académicas, presentaciones, organización de fiestas tradicionales semejantes a las de algún país iberoamericano y otras actividades del agrado de los jóvenes estudiantes. Esta estrategia fue identificada en las observaciones y en los testimonios brindados por los estudiantes que respondieron al cuestionario, en los que manifiestan sentir mucho interés por conocer otras culturas. Los motiva muy especialmente compartir la celebración de las fiestas tradicionales de España y países de América Latina con estudiantes de esos países, conocer su cultura juvenil, su música, sus danzas y sus actitudes ante la vida: "Organizamos muy a menudo diferentes fiestas. Durante éstas conocemos mejor la cultura de España y de la América Latina, por ejemplo Navidad y el Día de los Muertos (A2)”. [1]

c) Estrategia de aprendizaje autónomo del estudiante: las actividades desarrolladas exigen que los alumnos desarrollen sus habilidades de planificar, controlar y autorregular su propio aprendizaje. En las observaciones, profesores y alumnos comparten responsabilidades en la planificación, ejecución y monitoreo de las actividades académicas: "los estudiantes buscan material adicional, en fuentes bibliográficas y temas interesantes en las últimas noticias, para discutir" (D1). Cada estudiante o grupo de estudiantes asume compromisos en las actividades académicas dentro del aula, y en las actividades extracurriculares o de extensión universitaria, quedando a su cargo la planificación, la preparación de la actividad, la ejecución, el monitoreo y la presentación de un informe sobre la actividad realizada: "Organizamos fiestas con los estudiantes de otros países" (A3).

d) Estrategia de colaboración: los alumnos aprenden a trabajar en equipo, a buscar una comprensión mutua entre interlocutores y a tomar decisiones en conjunto mediante el desarrollo de tareas grupales: "Los alumnos asumen la responsabilidad de ejecutar tareas en equipo, como las que realizan para el Club Aula Hispánica, o el acompañamiento de visitantes extranjeros” (D2). Esta estrategia fue aplicada en la organización de todas las actividades, en las que los equipos de alumnos fueron los protagonistas principales. Esta forma de encarar el aprendizaje, además de fortalecer las habilidades de la dimensión metacognitiva, contribuye a despertar la motivación de los estudiantes, específicamente al fortalecer la subdimensión del interés extrínseco o social y su relación con las otras subdimensiones: interés intrínseco y capacidad de autorregular la propia motivación. 
e) Estrategia de construcción contextualizada: comprende acciones extensivas al contexto universitario y ciudadano que movilizan a la comunidad local, nacional e internacional generando un proceso de construcción social de la inteligencia cultural. La universidad actúa, con esta estrategia, como un poderoso motor que impulsa el desarrollo de la inteligencia cultural en su entorno. En las observaciones, se identificó esta estrategia en la planificación y ejecución de actividades, tanto internas como de extensión universitaria: “Tenemos aquí el Club Aula Hispánica, abierto al público, en el marco del cual hacemos las actividades culturales, literarias, etcétera"(D3). Se trata de actividades en las que participan con mucho entusiasmo estudiantes de otros centros, carreras o universidades, personas amigas, familiares y público en general. Se presentan y debaten en ellas temas atractivos de interés cultural y general que contribuyen a la contextualización de los saberes y a la formación de una cultura ciudadana favorable al diálogo intercultural. "Los alumnos, junto con estudiantes extranjeros de otras carreras, realizan jornadas interculturales en las escuelas de la ciudad" (D1). Estas jornadas sobre la cultura de cada país iberoamericano presentan las características, costumbres, danzas, música y otras tradiciones de cada país, junto con estudiantes o visitantes originarios de ese país. Los niños, maestros y familiares que asisten a estas jornadas tienen la oportunidad de conocer de cerca nuevas culturas y poder compartir un encuentro con personas que pertenecen a diferentes culturas.

\subsection{Actividades didácticas aplicadas para el desarrollo de la inteligencia cultural}

Las actividades didácticas mencionadas por los profesores entrevistados se clasificaron según el local en que se realizan, las personas que participan y el origen de los datos obtenidos (menciones de profesores o de alumnos). Los lugares en los que se ejecutan las actividades comprenden tanto locales internos al Centro Hispano-Ruso (aulas, auditorios, biblioteca), como también externos (biblioteca municipal, calles, museos, bulevares, escuelas y espacios públicos de la ciudad de Rostov del Don). En cuanto a los participantes, las actividades se clasifican en $a$ ) formación de profesores, $b$ ) aprendizaje para los estudiantes y $c$ ) extensión universitaria en las que intervienen los estudiantes, los profesores y el público en general.

Para fortalecer la formación de los profesores, ellos mencionaron que se realizan en el Centro HispanoRuso dos congresos internacionales de hispanistas por año, además de conferencias virtuales y presenciales. Los estudiantes mencionaron que se llevan a cabo constantemente conferencias internacionales, foros estudiantiles, charlas académicas interactivas, presentación de películas sobre la cultura hispánica y el club Aula Hispánica, que cuenta con la participación de estudiantes rusos y latinoamericanos. Las actividades didácticas en aula, según manifestaron los profesores, comprenden clases de español, cultura hispánica, historia de los pueblos hispanohablantes, traducción socio-política, entre otras. Estos datos aportados por los profesores fueron contrastados con las observaciones y los datos aportados por los alumnos, que además agregaron algunas actividades extracurriculares, como el acompañamiento a visitantes hispanohablantes, en calidad de intérpretes y guías, en los sitios turísticos y culturales de Rostov del Don y su entorno. La tabla 1 presenta una síntesis integrada de las actividades didácticas orientadas al desarrollo de la inteligencia cultural. 
TABLA 1

Actividades didácticas orientadas al desarrollo de la inteligencia cultural

\begin{tabular}{lllc}
\hline Actividad Académica & \multicolumn{1}{c}{ Lugar de ejecución } & \multicolumn{1}{c}{ Participan } & Mencionada por \\
\hline Congresos internacionales de hispanistas & Centro Hispano-Ruso & Profesores & Profesores \\
Conferencias virtuales & $\begin{array}{l}\text { Sala de conferencias } \\
\text { virtuales }\end{array}$ & Profesores y alumnos & Profesores \\
Conferencias presenciales & Centro Hispano-Ruso & Profesores y alumnos & Profesores \\
Fiestas tradicionales hispanoamericanas & Centro Hispano-Ruso & Profesores y alumnos & Alumnos \\
Foros estudiantiles & Centro Hispano-Ruso & Alumnos & Alumnos \\
Charlas académicas interactivas & Centro Hispano-Ruso & Alumnos & Alumnos \\
Películas sobre la cultura hispánica & Centro Hispano-Ruso & Alumnos & Alumnos \\
Lectura e investigación & Biblioteca de CHR y & Alumnos & Profesores y \\
& Biblioteca de Rostov & & alumnos \\
Discusión de textos literarios & Centro Hispano-Ruso & Profesores y alumnos & Alumnos \\
Acompañamiento como intérprete y guía de & Del Don Rostov y su & Alumnos & Alumnos \\
$\begin{array}{l}\text { actividades turísticas y culturales de } \\
\text { visitantes extranjeros }\end{array}$ & entorno & & Profesores, alumnos y \\
Club aula hispánica & Centro Hispano-Ruso & Profesores y \\
alumnos
\end{tabular}

Fuente: elaboración propia

\section{4. La visión de los profesores y estudiantes}

El análisis de los aportes de las estrategias observadas para el desarrollo de la inteligencia cultural, manifestados por profesores y estudiantes, se organizó en cuatro subcategorías, correspondientes a las cuatro dimensiones de la inteligencia cultural identificadas por Van Dyne et al. (2012): a) dimensión metacognitiva, $b$ ) dimensión cognitiva, $c$ ) dimensión motivacional y $d$ ) dimensión conductual.

\section{4.1. Dimensión metacognitiva}

En la visión de los profesores, las estrategias y actividades didácticas implementadas promueven progresivamente en los estudiantes el desarrollo de las habilidades de planificación, la conciencia durante la ejecución de las actividades y su monitoreo: "Los estudiantes organizan la celebración de fiestas tradicionales, foros, charlas interculturales en escuelas y otras actividades" (D2). Reforzando estos testimonios de los profesores, las observaciones muestran que los alumnos tienen un rol activo en la planificación de las actividades, que les permite desarrollar continuamente la conciencia necesaria para participar de forma crítica en encuentros interculturales, así como la capacidad de dar seguimiento de la dinámica y los cambios que surgen en estos encuentros: "Es interesante conocer las fiestas tradicionales de los pueblos iberoamericanos, y para eso organizamos fiestas con estudiantes de otros países"(A5). "Es muy lindo, pero esto está prohibido aquí" (A7, refiriéndose a la promoción de bebidas alcohólicas en un vídeo sobre fiestas de Andalucía, España). "Siento que desarrollo mis habilidades para interactuar con otras culturas al participar en la organización de la fiesta de la Navidad, y la fiesta del Día de los Muertos, con estudiantes de España y México" (A11). "Las 
mejores actividades son las que hacemos fuera del aula, pues los organizadores somos los estudiantes, entre nosotros" (A12).

\section{4. 2. Dimensión cognitiva}

Los alumnos adquieren gradualmente los conocimientos generales y específicos necesarios para la comunicación intercultural mediante actividades teórico-prácticas en las que se aplica el innovador método de galaxia espiral (Sémikova et al., 2015). En cuanto a las dificultades que enfrentan, los profesores señalaron: "A los alumnos les resulta difícil la búsqueda de información en fuentes bibliográficas, de material adicional al que proporciona el profesor" (D1). Mencionaron que los alumnos prefieren realizar sus búsquedas y revisiones en internet, antes que acudir a la biblioteca de la universidad, o a la biblioteca de la ciudad que cuenta con material bibliográfico en idioma español, en su departamento de lenguas extranjeras. Sin embargo, los alumnos manifestaron que sienten dificultades al realizar investigaciones por la escasez de libros en español en las bibliotecas, y que les gustaría contar con una literatura más abundante sobre la cultura de los pueblos iberoamericanos: "La biblioteca sólo tiene un ejemplar de algunos libros importantes, y a veces ni siquiera eso" (A5).

Otro de los desafíos que enfrentan los estudiantes en esta dimensión, señalada por los profesores, se refiere al conocimiento y la discusión de temas de actualidad, sociales, políticos, económicos o culturales: "Les cuesta participar en los debates porque no conocen los temas de actualidad" (D1). Contrastando con esta visión de sus profesores, los alumnos manifiestan que su principal dificultad se encuentra al inicio de los debates, sienten falta de autoconfianza para empezar a hablar, temor que van perdiendo de forma gradual a medida que son animados a expresarse por compañeros y profesores.

\section{4. 3. Dimensión motivacional}

En la visión de los profesores y estudiantes las actividades que despiertan mayor interés por parte de los estudiantes son las charlas académicas, los foros estudiantiles, la celebración de las fiestas navideñas y las conferencias de estudiantes y profesores extranjeros visitantes. Los estudiantes mencionaron que se sienten muy motivados por las fiestas organizadas con estudiantes de otros países. Celebran con ellos la Navidad, el Día de los Muertos y otras fiestas tradicionales de países iberoamericanos. El Centro Hispano-Ruso recibe constantemente a estudiantes españoles de la Universidad de Cádiz, que vienen a realizar una pasantía gracias a las becas de movilidad estudiantil que otorgan empresas y organismos internacionales.

Los estudiantes mencionaron que se sienten motivados por las conferencias interculturales presenciales con profesores extranjeros, las videoconferencias con estudiantes de universidades de América Latina y España (mencionaron las charlas con México y Valladolid) y las lecciones con profesores extranjeros que incluyen conversación y debate. "Me gustan las actividades relacionadas con la historia, las tradiciones y costumbres" (A15), menciona un estudiante. Los motiva muy especialmente, según testimonios de otros estudiantes, el "debate de la situación actual en el mundo" (A8), los temas de "la cultura de otros pueblos" (A10) o los “temas de actualidad” (A14).

El Club Aula Hispánica es una de las actividades señaladas como de mayor interés por los estudiantes. Esta actividad de extensión universitaria acrecienta la motivación extrínseca o social de los estudiantes porque pueden invitar a los familiares y amigos, que con frecuencia participan. El reconocimiento social, la apertura hacia otras culturas y el quiebre de las barreras que impiden el diálogo intercultural se extiende a la ciudad de Rostov del Don y su entorno mediante esta actividad de extensión universitaria en la que los ciudadanos tienen oportunidad de aproximarse a la cultura iberoamericana en forma directa y de derribar sus prejuicios. 


\section{4. 4. Dimensión conductual}

Conforme señalan los testimonios de los estudiantes y profesores participantes, todas las actividades observadas en este estudio promueven el desarrollo de conductas y formas de actuar favorables a la comunicación intercultural. A medida que se desarrollan las habilidades de comunicación verbal en español, se desarrollan también las habilidades de comunicación no verbal y la capacidad de autorregulación de la comunicación: "Es un poco difícil hablar con la gente de diferentes países durante las actividades. Sin embargo, esto es muy interesante y muy importante" (A16). Otro estudiante señala que siente mayor dificultad en "las actividades con los estudiantes de América Latina porque a veces la variante de su país es un poco difícil para entender" (A18).

Entre las actividades que promueven las habilidades de comunicación verbal, comunicación no verbal y autorregulación de la comunicación, los estudiantes mencionan como una de las más efectivas a la actividad de acompañar a los extranjeros hispanohablantes porque les exige un mayor esfuerzo en la selección de recursos comunicativos verbales y no verbales. La actividad se realiza en pareja o pequeños grupos (dos o tres estudiantes) que planifican el recorrido, marcan los encuentros, acompañan a visitantes extranjeros y actúan en calidad de guías e intérpretes de lo que observan en el recorrido. En esa actividad, los alumnos deben ejercer el rol de puente comunicacional entre la cultura del visitante y la cultura de la comunidad local, y ese puente requiere de una selección cuidadosa de los recursos comunicativos que se aplicaron para hacer posible una conexión real entre ambas culturas. En ese desafío, los estudiantes desarrollan de forma intuitiva y práctica su capacidad de autorregulación en el proceso de selección de los recursos comunicacionales y sus posibilidades de aplicación, así como también la habilidad de contornar las barreras interculturales que pudieran presentarse en cada situación.

\section{AnÁlisis Prospectivo: aplicabilidad en las universidades del Paraguay}

A partir de los resultados obtenidos, este artículo tiene su aporte dirigido a nuevos conocimientos que podrán influir, en el futuro, en el diseño de estrategias didácticas para el desarrollo de la inteligencia cultural en las universidades de Paraguay. Las cinco estrategias didácticas identificadas en este estudio (estrategia comunicativa, motivación del estudiante, autonomía del estudiante, aprendizaje colaborativo y construcción colectiva contextualizada) pueden servir de referencia para el diseño de estrategias innovadoras que promuevan el desarrollo de la inteligencia cultural en las universidades del Paraguay.

A pesar de las diferencias culturales, económicas y sociales que existen entre las universidades paraguayas y la Universidad Federal del Sur, las cinco estrategias mencionadas son aplicables siempre que la comunidad universitaria considere prioritaria la internacionalización de la educación superior y la promoción del diálogo intercultural entre los pueblos.

También pueden servir de referencia las actividades didácticas efectuadas en el Centro Hispano-Ruso, identificadas en este estudio: conferencias internacionales, conferencias virtuales o videoconferencias, celebración de fiestas interculturales, foros estudiantiles, discusiones literarias y charlas interactivas con momentos de debate. Algunas de estas actividades ya son aplicadas habitualmente por universidades del Paraguay, como las conferencias internacionales, en tanto que otras no son frecuentes o ni siquiera forman parte de las actividades universitarias. Salvo las conferencias virtuales, que requieren salas especiales con equipamiento informático adecuado, las demás actividades no requieren grandes inversiones ni cambios estructurales. Su aplicabilidad depende en exclusivo de la voluntad de los miembros de la comunidad educativa universitaria, independiente de los recursos financieros disponibles.

La recepción de estudiantes y profesores extranjeros en muchas de las universidades paraguayas, mediante programas de movilidad estudiantil o docente, es poco frecuente. Si bien se cuenta con vinculaciones y 
programas de becas que pueden apoyar esta movilidad, las universidades paraguayas no priorizan la gestión eficiente de estos recursos ni actúan de forma favorable para este tipo de intercambio. El diseño de programas interculturales con actividades atractivas para estos estudiantes y profesores extranjeros generaría un flujo mayor de estudiantes y docentes extranjeros que elijan sus pasantías en universidades de Paraguay.

Algunas universidades paraguayas reciben un número considerable de estudiantes en cursos regulares de grado y posgrado provenientes de los países limítrofes gracias a los acuerdos regionales que facilitan el reconocimiento de los títulos. Sin embargo, las universidades no siempre promueven actividades de integración cultural con estos estudiantes, que muchas veces son segregados en cursos exclusivos para ellos. El diseño de estrategias y actividades integradas que propicien la comunicación intercultural puede favorecer el desarrollo de la inteligencia cultural en la comunidad educativa universitaria, y así contribuir al fortalecimiento de una actitud de apertura hacia otras culturas, además de propiciar el desarrollo de la inteligencia cultural.

Las actividades de extensión universitaria identificadas en este trabajo también pueden servir de referencia para el diseño de actividades similares en las universidades del Paraguay. Las características del Club Aula Hispánica, con exposiciones de profesores y estudiantes extranjeros, complementadas por demostraciones, filmes, música y debates sobre aspectos culturales de su país, pueden replicarse en las universidades de Paraguay mediante la organización de clubes culturales internacionales o específicos de determinados países. Las charlas interculturales en las escuelas de la ciudad también pueden servir de referencia para el diseño de jornadas interculturales de extensión universitaria, de modo que las universidades paraguayas contribuyan a la promoción de una conciencia social favorable a la comunicación intercultural en el país.

\section{CONSIDERACIONES FINALES}

El artículo presentado identificó un conjunto de estrategias didácticas innovadoras para el desarrollo de la inteligencia cultural aplicado en el Centro Hispano-Ruso de la Universidad Federal del Sur mediante las observaciones recabadas y los testimonios aportados por estudiantes y profesores. Por tratarse de un estudio de caso, de nivel exploratorio, los resultados obtenidos no conducen a la afirmación de conclusiones que puedan aportar nuevos conocimientos generales sobre el tema. Con estas limitaciones, sin embargo, los resultados permiten sistematizar algunas consideraciones o aproximaciones para una mejor comprensión del objeto de estudio y su posible proyección en el diseño de estrategias didácticas innovadoras que promuevan la internacionalización de la educación superior en América Latina.

De acuerdo con los resultados obtenidos mediante las observaciones realizadas en el Centro HispanoRuso, las estrategias didácticas innovadoras que promueven el desarrollo de la inteligencia cultural son a) enfoque comunicativo, $b$ ) motivación del estudiante, $c$ ) autonomía del estudiante, $d$ ) aprendizaje colaborativo y $e$ ) construcción colectiva contextualizada. Las cinco estrategias se implementan mediante actividades didácticas y de extensión universitaria; se clasificaron según el local de realización, los participantes y la fuente de información. Las actividades didácticas realizadas en el local del Centro Hispano-Ruso incluyen conferencias internacionales, conferencias virtuales o videoconferencias, celebración de fiestas interculturales, foros estudiantiles, discusiones literarias y charlas interactivas con momentos de debate. Los profesores, a su vez, cuentan con congresos, conferencias y videoconferencias internacionales específicas para ampliar su formación. La movilidad estudiantil y la presencia constante de profesores y estudiantes hispanohablantes, facilitada por la articulación de vinculaciones con universidades de la región iberoamericana, amplía la actitud de apertura y desarrolla las habilidades comunicativas interculturales de los estudiantes.

Las actividades de extensión universitaria observadas fueron el Club Aula Hispánica y las charlas interculturales en las escuelas de la ciudad. Estas actividades contribuyen a desarmar las barreras culturales 
presentes en la sociedad rusa, específicamente en la ciudad de Rostov del Don, y a facilitar la construcción social de una cultura que favorezca la comunicación intercultural.

A partir de los resultados de las observaciones y los testimonios de los estudiantes y profesores participantes, se concluye que las estrategias y actividades observadas brindan aportes significativos al desarrollo de las cuatro dimensiones de la inteligencia cultural señaladas por Van Dyne et al. (2012): dimensión cognitiva, dimensión metacognitiva, dimensión de la motivación y dimensión conductual. Las vinculaciones y los sistemas de intercambio de profesores y alumnos con universidades de España y América Latina cumplen un papel estratégico al promover la comunicación intercultural presencial y virtual. De esta forma, la universidad cumple su rol integrador de culturas, que se afirma como el motor que dinamiza el desarrollo social de la inteligencia cultural a nivel local y extiende su influencia a nivel regional, y proyecta al Centro Hispano-Ruso como un centro de referencia a nivel nacional e internacional.

Tomando en cuenta estas consideraciones, se sugiere aplicar estas actividades didácticas en el diseño de estrategias para la internacionalización efectiva de las universidades del Paraguay, y también en universidades de otros países de América Latina. Se recomienda, además, dar continuidad a esta línea de investigación a través de la promoción de futuros estudios que incluyan la adecuación y el diseño de estas estrategias, y su implementación en el contexto universitario paraguayo o por medio de estudios comparativos sobre su diseño, implementación y resultados obtenidos.

\section{ReFERENCIAS}

Bruner, J. (2012). What psychology should study? International Journal of Educational Psychology, 1, 5-13.

Canese, M. (2010). Internacionalización de la Educación Superior. Estudio comparativo realizado en la República de China (Taiwán). Disponible en https://repositorio.ufsc.br/xmlui/handle/123456789/96788

Centro Hispano-Ruso. (2016) Portal del CHR: sobre el Centro. Disponible en http://esp-centr.sfedu.ru/es/

Domingo Depaula, P. y Celeste Azzollini, S. (2012). Inteligencia cultural, valores y motivación para el aprendizaje en estudiantes militares argentinos. Revista de Psicología (PUCP), 30(1), 75-102.

Earley, P. C. y Ang, S. (2003). Cultural intelligence: Individual interactions across cultures. Stanford: Stanford University Press.

Earley, P. C. y Mosakowski, E. (2004). Cultural intelligence. Harvard business review, 82(10), 139-146.

Esteban-Guitart, M. (2012). Del Homo intellectualis al Homo educandus. La hipótesis de la inteligencia cultural y la intencionalidad compartida. Boletin de Psicología, 105, 23-42.

Esteban-Guitart, M., Vila, I. y Ratner, C. (2013). El carácter macrocultural de la identidad nacional. Estudios de Psicología, 34(1), 27-35.

Hernández Sampieri, R., Fernández Collado, C. y Baptista Lucio, P. (2014). Metodología de la investigación (sexta edición). México: McGraw-Hill.

Mora, R. J. F. (2010). Orientaciones básicas para el diseño de estrategias didácticas. Tendencias pedagógicas, 16, 221-236.

Sémikova, M. E., Karpóvskaya, N. y Korman, E. A. (2015). Aplicación de estrategias didácticas innovadoras en la enseñanza del español como lengua extranjera, en La identidad nacional a través del diálogo entre culturas: estudios cientificos sobre el área de las humanidades en los espacios cientificos ruso e iberoamericano. Tomo 2. Rostov del Don: Editorial de la Universidad Federal del Sur.

Van Dyne, L., Ang, S., Ng, K. Y., Rockstuhl, T., Tan, M. L. y Koh, C. (2012). Subdimensions of the four factor model of cultural intelligence: Expanding the conceptualization and measurement of cultural intelligence. Social and personality psychology compass, 6(4), 295-313.

Vigotsky, L. y Luria, A. (2007). El instrumento y el signo en el desarrollo del niño. Madrid: Fundación Infancia y Aprendizaje.

Vigotsky, L. (1979). El desarrollo de los procesos psicológicos superiores. Barcelona: Crítica. 
Notas

[1] Fiesta del Día de los Muertos: fiesta tradicional mexicana de origen precolombino que se celebra el 2 de noviembre. CC BY-NC-ND 